\title{
Regulation of Antigen Export to the Cytosol During Cross-Presentation
}

\author{
Marine Gros and Sebastian Amigorena* \\ INSERM U932, Institut Curie, Paris, France
}

Cross-priming refers to the induction of primary cytotoxic $\mathrm{CD}^{+} \mathrm{T}$ cell responses to antigens that are not expressed in antigen presenting cells (APCs) responsible for $\mathrm{T}$ cell priming. Cross-priming is achieved through cross-presentation of exogenous antigens derived from tumors, extracellular pathogens or infected neighboring cells on Major Histocompatibility Complex (MHC) class I molecules. Despite extensive research efforts to understand the intracellular pathways involved in antigen cross-presentation, certain critical steps remain elusive and controversial. Here we review recent advances on antigen cross-presentation, focusing on the mechanisms involved in antigen export to the cytosol, a crucial step of this pathway.

Keywords: dendritic cells, cross-presentation, cytosolic antigen export, ERAD, endosomal leakage

\section{INTRODUCTION}

OPEN ACCESS

Edited by:

Diana Dudziak,

Universitätsklinikum Erlangen,

Germany

Reviewed by:

Marianne Boes,

Utrecht University, Netherlands

Margarita Del Val,

Spanish National Research Council

(CSIC), Spain

*Correspondence:

Sebastian Amigorena

sebastian.amigorena@curie.fr

Specialty section:

This article was submitted to

Antigen Presenting Cell Biology,

a section of the journal

Frontiers in Immunology

Received: 29 September 2018 Accepted: 09 January 2019

Published: 28 January 2019

Citation:

Gros M and Amigorena S (2019) Regulation of Antigen Export to the Cytosol During Cross-Presentation. Front. Immunol. 10:41.

do: 10.3389/fimmu.2019.00041
Dendritic cells (DCs) play a central role in immune homeostasis by linking innate sensing to adaptive immune responses. After sampling antigens in peripheral tissues, DCs mature and migrate to lymph nodes, where they initiate adaptive immune responses by presenting processed antigens in the context of Major Histocompatibility Complex (MHC) molecules to T cells. For a long time, the generally accepted paradigm supposed that exogenous antigens were exclusively presented via MHC-II molecules to $\mathrm{CD} 4^{+} \mathrm{T}$ cells, while endogenous cytosolic antigens, derived from self or foreign proteins, were loaded on MHC-I, thereby leading to naïve cytotoxic $\mathrm{CD}^{+} \mathrm{T}$ cell activation. Yet, this simple assumption failed to explain how cytotoxic immune responses could be mounted against pathogens that do not readily infect DCs. This apparent contradiction was resolved by the discovery of cross-presentation, a process enabling the delivery of exogenous antigens to the MHC-I pathway for cross-priming $\mathrm{CD}^{+}$cytotoxic T cell responses $(1,2)$. Since its first description over forty years ago, our understanding of the sequence of events governing antigen cross-priming has extensively increased, leading to the description of two main pathways of antigen cross-presentation, referred to as "vacuolar" and "cytosolic." While the requirement for cross-presentation in the initiation of anti-tumor immune responses is now well established (3-7), its control and the precise intracellular routes involved remain incompletely understood and, for some parts, controversial.

Here, we review the most recent advances in the analysis of antigen cross-presentation in mouse (unless stated otherwise), with a particular emphasis on the advances in understanding of antigen export to the cytosol, a crucial, yet debated, step of the cytosolic pathway.

\section{PATHWAYS FOR ANTIGEN CROSS-PRESENTATION}

In 1976, seminal work by M. Bevan showed that exogenous antigens could be presented on MHC-I molecules and prime cytotoxic immune responses, thereby unearthing a novel antigen presentation pathway that he called cross-priming $(1,2)$. However, the molecular mechanisms underlying cross-priming and "cross-presentation" remained elusive until the early nineties. 
At that time, several lines of evidence reported that cross-presentation of bacterial antigens [i.e., the 257-264 $\mathrm{H}-2 \mathrm{~K}^{\mathrm{b}}$-restricted epitope of ovalbumin (OVA) fused to E. coli $\mathrm{Crl}$ protein] was resistant to proteasome inhibitors (8) (suggesting lysosomal processing of the corresponding peptides), unaffected by brefeldin A (BFA) treatment (8-10) [arguing against a critical role for endoplasmic reticulum (ER)-Golgi transport] and most of the time, occurred independently from TAP, the transporter mediating peptide import into the $\operatorname{ER}(8,11)$. These observations led to the first description of the "vacuolar pathway." After internalization, antigens remain confined in intracellular compartments, where they undergo lysosomal degradation, a process largely dependent on cathepsin S activity (12), and followed by loading onto post-Golgi MHC-I molecules.

Simultaneous studies with particulate, non-bacterial antigens (i.e., bead-bound OVA), showed that TAP1 deficiency in macrophages, as well as BFA treatment, abolished their ability to cross-present exogenous antigens, thereby suggesting that antigen-derived peptides must be transferred from the cytosol to the ER to bind newly synthesized MHC-I molecules (13). Additionally, cross-presentation was disrupted by proteasome inhibitors (13-16), consistent with a model in which antigens are delivered into the cytosol before proteasomal degradation and peptide import into the ER. This pathway, later termed the "cytosolic pathway," implies the export of antigens from endocytic compartments to the cytosol. The first experimental evidence of this crucial step was provided by the use of gelonin, a membrane-impermeant toxin that inactivates ribosomes when transferred to the cytosol. Macrophages phagocytosing gelonincoated beads displayed reduced protein synthesis, indicating export of bead-bound gelonin to the cytosol $(13,14)$.

The aforementioned pivotal studies used mouse macrophages as models of antigen-presenting cells (APCs). It later became clear that DCs, rather than macrophages, cross-present antigens and cross-prime cytotoxic immune responses efficiently (17, 18), by means of different properties of their phagocytic pathway, including lower degradation capacity (19). When considering DCs, these cells represent a series of ontogenically and functionally diverse populations. In mice, two main resident DC subsets are found in the spleen and lymph nodes, namely Batf3-dependent $\mathrm{CD} 8^{+} \mathrm{XCR}^{+}$DCs (DC1s) and IRF4dependent $\mathrm{CD}^{-} \mathrm{CD}^{-} 1 \mathrm{~b}^{+}$DCs (DC2s) [reviewed in (20)]. At steady state, DC1s cross-present cell-associated antigens more efficiently than their DC2 counterparts, a capacity first attributed to their increased ability to capture this type of antigen $(21,22)$. Later experiments showed that higher crosspresentation efficacy in mouse DC1s is intrinsic and unrelated to the route of antigen uptake $(23,24)$, thus contrasting with the Fc $\gamma$ R-dependent optimization of cross-presentation observed in human DC1s (25). In mouse, surface receptors, including Clec9A/DNGR-1 (26-29) or mannose receptor (MR) (30), were proposed to preferentially deliver antigens to the crosspresentation pathway, most likely through delaying delivery of their cargoes to late endosomal and lysosomal degradative compartments. DC1s also bear specialized endocytic properties that reduce/delay acidification and degradation of endocytic cargo $(19,31)$.
Consistent with these in vitro observations, mice deficient for DC1s (5), or displaying cross-presentation-defective DCs $(4,6)$, fail to mount cytotoxic immune responses against tumors and to control tumor development, even after treatment with checkpoint blockers. Although DC1s are best suited for crosspresentation both in vitro and in vivo, DC2s' ability to crosspresent is increased by targeting antigens to DC2 specific receptors, such as Fc $\gamma \mathrm{R}$ (32) or DCIR2, in a stimulatory context (33), thus suggesting that both $\mathrm{DC} 1$ and $\mathrm{DC} 2$ are capable of cross-presenting antigens depending on the conditions.

The relative contributions of the cytosolic and vacuolar pathways to in vivo cross-presentation and cross-priming remain unclear. TAP dependency can potentially affect both pathways, as it impairs the exit of MHC-I molecules from the ER (34-37). Whether critical players in cross-presentation, such as Sec22b $(4,38,39)$ or Rab43 (40), which are both required for effective cross-priming, are selectively involved in one or both pathways is unknown. The best available evidence for the cytosolic pathway being predominant in cross-priming comes from a study using mice defective for the immunoproteasome subunit LMP7. These mice show impaired cross-priming for an immunoproteasome-dependent $\mathrm{H}-\mathrm{Y}$ epitope, supporting a critical role for proteasome-dependent processing, and therefore, for the cytosolic pathway in vivo (41). Since delivery of internalized antigens to the cytosol is very ineffective in most cell types, DCs might have developed specialized pathways to link these two subcellular compartments.

\section{BIOLOGICAL PARAMETERS INFLUENCING ANTIGEN EXPORT TO THE CYTOSOL \\ Nature of Cytosolic Export-Competent Cells}

By using gelonin activity or cytosolic fluorescence quantification as readouts, initial studies showed that inflammatory (14) or activated (16) mouse macrophages displayed a measurable ability to export bead-conjugated (14) or soluble (16) cargo into the cytosol. Further work revealed that soluble or complexed antigens also get access to the cytosol in steadystate bone-marrow derived DCs (BMDCs) or in a DC cell line, without prior activation $(17,18)$. Moreover, antigen export to the cytosol is more efficient in DCs than in macrophages, as illustrated by subcellular fractionation and subsequent western blotting (18). To assess whether DC subsets differ in their capacity to perform such transfer, Lin et al. developed a cytochrome c-based assay relying on the selective apoptosis of cells exporting exogenous cytochrome c into the cytosol (42). Only a fraction of DC1s showed susceptibility to cytochrome c-induced apoptosis, indicating a functional specialization for endosome to cytosol transport in these cells $(42,43)$. Notably, this cytochrome c-sensitive DC1 population strictly corresponds to the cohort of efficient crosspresenters, whereas cytochrome c-resistant DC1s cross-present antigens inefficiently and share other functional features with DC2s (42). 


\section{Nature of Antigens Exported to the Cytosol}

Early microscopy observations showed that fluorescent (i.e., dextrans) or soluble (i.e., enzymatically active horseradish peroxidase: HRP) antigens gained access to the cytosol in DCs $(17,18)$. While $3-40 \mathrm{~K}$ dextrans are rapidly relocated to the cytosol, higher molecular mass dextrans (500-2,000 K) remain vacuolar (18), suggesting that antigen export to the cytosol is size-selective $(18,44)$. Particulate antigens, which are more efficiently cross-presented than soluble ones (14), often form large aggregates and therefore require dissociation before their translocation to the cytosol. Indeed, inhibition of vacuolar acidification abolishes the disaggregation of immune complexes and their subsequent cytosolic export (18), thus pointing to a crucial role of slightly acidic endo/phagosomal $\mathrm{pH}$ in this process. While some degree of degradation might favor antigen export to the cytosol due to the size-restriction of transported antigens (18), high proteolytic activity, favored by acidic $\mathrm{pH}$, could destroy MHC-I-binding epitopes. In this regard, regulation of endocytic $\mathrm{pH}$ is of crucial importance. In DCs' endocytic compartments, incomplete assembly of v-ATPase proton pump together with Rab27a-dependent recruitment of NOX2 jointly lead to active alkalinization of luminal $\mathrm{pH}(19,45)$, thereby preserving antigens from detrimental excessive degradation (46).

\section{Export to the Cytosol and DC Activation}

Aside from putative intrinsic properties of DC1s, extrinsic signals, such as Toll-Like Receptor stimulation, influence antigen export to the cytosol. Indeed, short (3-5 h) lipopolysaccharide (LPS) stimulation of BMDCs increases the proportion of cells displaying exogenous HRP in their cytosol (47). A possible explanation for the observed LPS-mediated increase in antigen export may reside in the requirement for TRIF in this process (48). Until recently, absence of quantitative reliable antigen export assays based on endotoxin-free reagents impeded detailed analysis of the role of DC activation in antigen transport to the cytosol. Recently published export assays should overcome this limitation (49).

\section{Kinetics of Antigen Export to the Cytosol in DCs}

Kinetics studies showed that HRP appeared in BMDC cytosol only $15 \mathrm{~min}$ after internalization (17). Rapid egress suggests that antigens are exported from early endosomes (50), as supported by microscopy experiments (51) or by mathematical modeling (52). The latter predicts that $20 \mathrm{~min}$ after internalization, cytosolic export of yeast-derived antigen competes with degradation associated with maturation of the endocytic compartment. Thus, only a tiny fraction of, at least, non-complexed antigens released after this time point might contribute to cross-presentation. Cytosolic translocation of HRP immune complexes appears after $60 \mathrm{~min}$, and reaches a plateau after $6 \mathrm{~h}$ (18). Similar findings were reported for cytosolic egress of antigens associated to beads $(53,54)$. Additionally, these two studies provided compelling evidence that ER-mediated delivery of MHC-I loading machinery to the phagosome rendered this compartment competent for cross-presentation (55) following TAP-mediated import of cytosolic peptides $(53,54)$. While the relative contributions of ER and plasma membrane to the formation of cross-presentationcompetent phagosomes remain debated $(39,56)$, Houde et al. postulated that an ER transporter, Sec61, might be involved in the translocation of antigens from the phagosomal lumen to the cytosol (53). This hypothesis was later experimentally supported by several studies detailed in the next section.

\section{MOLECULAR MECHANISMS OF ANTIGEN EXPORT TO THE CYTOSOL}

\section{ERAD Transporter-Dependent Hypothesis}

Existence of a transporter mediating antigen export to the cytosol naturally imposes conformational constraints on the translocated antigen. Indeed, antigens are unlikely to be transported in their native structure, considering the narrow diameter of known transporter pores, and are therefore expected to undergo an unfolding step before translocation. Supporting this idea, fixed OVA is less efficiently translocated into the cytosol than structurally flexible one (57). Moreover, during unfolding, reduction of disulfide bonds by GILT, a phagolysosomal thiol reductase constitutively expressed in APCs, is essential for cytosolic export of viral disulfide-rich antigens and subsequent cross-presentation (58) (Figure 1, left panel).

Although the requirement for protein unfolding suggests that antigens gain access to the cytosol through a transporter, the nature of the channel mediating this process remains controversial. Studies attempting to answer this question reported interactions between unfolded OVA and members of the ER-associated degradation (ERAD) machinery in ER-associated compartments (59), consistent with previous findings $(53,54)$. This observation led to the hypothesis that the ERAD machinery, mediating retro-translocation of misfolded proteins from the ER lumen to the cytosol, potentially through the trimeric Sec61 channel, could also operate from endocytic compartments during cross-presentation. The first functional insights into ERAD contributions to antigen export to the cytosol, came from studies using exotoxin A (ExoA), a bacterial toxin binding to the cytosolic N-terminal domain of Sec61 $\alpha$, and resulting in channel closure $(60,61)$. ExoA treatment reversed the ICP47-mediated inhibition of TAP, the latter resulting from the translocation of exogenously delivered ICP47 to the cytosol and its subsequent interaction with the cytosolic side of TAP (62). This finding, associated with the observed decrease in OVA cross-presentation following ExoA treatment $(62,63)$ or siRNA-mediated silencing of Sec61 $(48,59)$, strongly pointed to Sec61 being the channel controlling antigen export to the cytosol (Figure 1, left panel). In line with this hypothesis, the expression of the Sec61 $\alpha, \beta$ and $\gamma$ subunits is increased in DC1s, as compared to DC2s, correlating with their specific cross-presenting ability (64).

However, it has been extremely difficult to address the precise contribution of Sec61 in antigen cross-presentation and retrotranslocation from endo/phagosomes, as this channel also mediates co-translational import of proteins, including MHCI, into the ER. To shed some light on this issue, Zehner et al. used a intrabody-based approach aiming to retain Sec61 in the ER and thereby prevent its recruitment to endocytic 


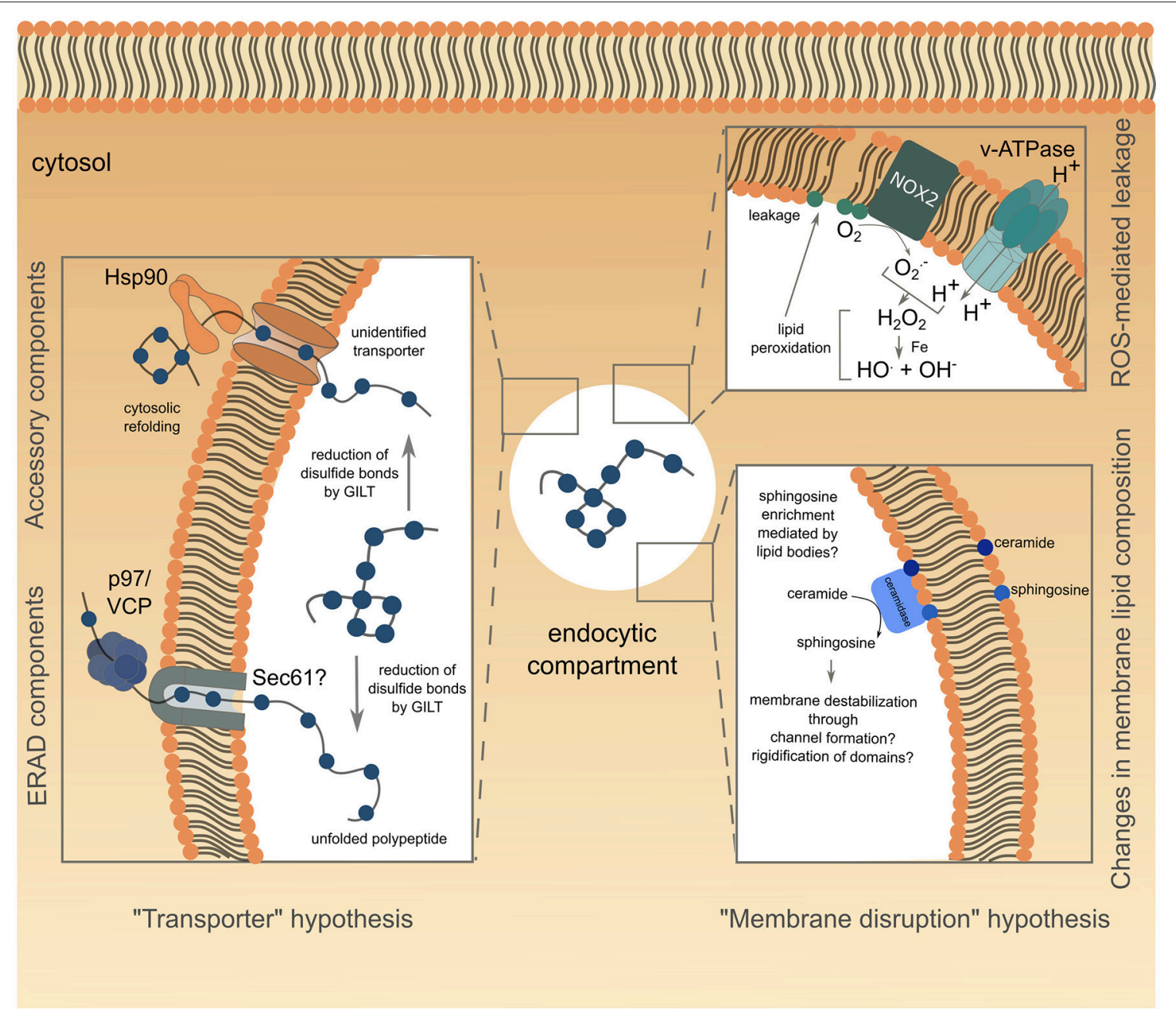

FIGURE 1 | Schematic representation of the current understanding of antigen export to the cytosol during cross-presentation. The transporter hypothesis is depicted on the left side of the figure, with complexes involved grouped according to known (for ERAD) or supposed (Hsp90 with unknown transporter) interactions. The alternative membrane disruption hypothesis is depicted on the right side of the figure. The ROS-mediated leakage part has been confirmed experimentally, while modification of endo/phagosome lipid composition, suggested by biophysical studies, is speculative and lacks functional relevance in antigen export to the cytosol.

compartments (48). Expression of the anti-Sec61 intrabody in BMDCs impairs antigen export to the cytosol and OVA crosspresentation, consistent with a role for Sec61 outside the ER, possibly in endosomes. Still, the involvement of Sec61 itself in ERAD-dependent retrotranslocation remains unclear and fraught with technical issues [reviewed in (65)]. Additionally, recent work has shown that sustained inhibition of Sec61 with a specific toxin, mycolactone, has no effect on antigen export to the cytosol, and indirectly reduces OVA cross-presentation through downregulation of other players in the pathway, including MHC-I (66). While Sec61 involvement in cytosolic antigen translocation needs further clarification, other ERAD components, such as Hrd1 and Derlin-1, might be alternative candidates.

Hrd1, an ER-resident ubiquitin ligase tagging ERAD substrates, exhibits six transmembrane domains, which is enough to form a channel $(67,68)$. siRNA-mediated depletion of
Hrd1 in DCs results in decreased antigen export to the cytosol and cross-presentation, as well as impaired MHC-II presentation (48). These alterations in antigen presentation pathways might be caused by Hrd1 silencing-mediated ER stress and therefore require further investigation. On the other hand, the protease Derlin-1 (Der1), comprising four transmembrane domains, cannot form a channel but could possibly function as an accessory subunit of the export channel (69) by trapping ERAD substrates and rerouting them for cytosolic degradation (70). Yet, antigen cross-presentation is not perturbed by Der1 silencing in both murine BMDCs (48) and human monocyte-derived DCs (71), thus excluding a putative role for Der1 in antigen export to the cytosol.

To date, the best evidence available suggests that ERAD might control antigen transfer to the cytosol through the activity of the AAA ATPase p97. P97 forms an hexameric ring and is thought to provide the energy necessary for passage of proteins through 
the retrotranslocating channel (72). Exogenous addition of p97 to isolated phagosomes loaded with luciferase leads to luciferase release, whereas addition of a dominant negative version of p97 fails to do so (62), suggesting a role for the ATPase in antigen translocation from phagosomes (Figure 1, left panel). Along the same lines, human and mouse DCs silenced for p97 $(59,71)$ or expressing a dominant negative form of p97 (62), display impaired cross-presentation of MelanA and OVA antigens, respectively, whereas p97 overexpression enhances this pathway (73). P97 is recruited to endosomes following mannose receptor (MR)-poly-ubiquitination. This post-translational modification proves to be crucial for antigen export to the cytosol and OVA cross-presentation as expression of a mono-ubiquitinated form of the MR is sufficient to reduce both processes (73). Of note, MR poly-ubiquitination is triggered by OVA binding to the receptor, and is negatively regulated by the ESCRT (Endosomal Sorting Complex Required for Transport)-I protein TSG-101 (73).

Several studies investigating the role of p97 in antigen export to the cytosol used the luciferase enzyme to monitor this intracellular event $(62,74)$. Following unfolding in endocytic compartments and subsequent translocation into the cytosol, luciferase would need to be refolded to exert its functionality, a process likely mediated by the chaperone Hsp90. Indeed, cytosolic refolding of exogenous unfolded luciferase is compromised in Hsp90 $\beta$-silenced human DCs or in DCs treated with the Hsp90 inhibitor radicicol (74). Furthermore, Hsp90 $\alpha$ deficiency not only inhibits cross-presentation in mouse BMDCs, but also decreases cytosolic translocation of OVA, therefore implying that Hsp90 itself could mediate antigen transport to the cytosol $(43,57)$. Additionally, Hsp90 could protect the exported antigens from premature cytosolic degradation, before Hsp70mediated targeting to the proteasome (57) (Figure 1, left panel).

The "transporter hypothesis" has, so far, garnered the most experimental support, as the main conduit for antigen export. However, it still raises important questions. Given the high degree of substrate selectivity during ERAD [reviewed in (65)], the use of a unique transporter translocating a wide variety of antigens seems unlikely. Moreover, this hypothesis fails to explain how large, non-protein molecules, such as dextrans, can be transferred to the cytosol in absence of ubiquitination, the latter being a prerequisite for ERAD-mediated translocation. Altogether, these observations do not exclude a role for ERAD in antigen export to the cytosol, but rather suggest the contribution of additional mechanisms.

\section{Alternative Hypothesis: Rupture of the Antigen-Containing Compartment}

The first descriptions of the cytosolic pathway for crosspresentation supposed that antigens could escape endocytic compartments through membrane rupture. This hypothesis, at that time termed "indigestion model," relies on the observation that large particles are more efficiently cross-presented than small ones, and could thus be responsible for phagosomal overload, leading to membrane disruption and efficient antigen leakage to the cytosol (14). Despite intensive use of this pathway for cytosolic delivery of antibodies or bioactive proteins conjugated with endosomolytic peptides (75-77), evidence of its contribution to cross-presentation were lacking, until recently.

\section{ROS, Lipid Peroxidation, and Membrane Rupture}

A recent study showed that following LPS stimulation, VAMP8dependent NOX2 recruitment to BMDC endosomes resulted in Reactive Oxygen Species (ROS) production and subsequent endosomal lipid peroxidation $(78,79)$. This alteration of lipid structure disrupts endosomal membrane integrity, leading to antigen escape to the cytosol and OVA cross-presentation (78) (Figure 1, upper right panel). Interestingly, ROS production in endocytic compartments is intrinsically linked to cells' crosspresenting ability. Indeed, DCs show sustained and stronger ROS production than macrophages (19), the latter subset increasing phagosomal ROS production, as well as cross-presentation, only after activation $(17,19)$. Moreover, ROS generation is higher in DC1 than in DC2 phagosomes, thereby correlating with the enhanced ability of DC1s to cross-present antigens (31). Biophysical studies provided mechanistic insights into lipid peroxidation-dependent membrane rupture. Oxidized lipid-rich artificial bilayers show higher water permeability (80), as well as increased membrane curvature, associated with micellization and membrane destabilization (81).

\section{Changes in Endolysosomal Membrane Lipid Composition}

Aside from lipid peroxidation, enrichment in ceramide, and to a greater extent in sphingosine, also triggers membrane permeability to solutes (82). While some studies proposed that sphingosine-based lipids could form large channels in membranes through an "all or none" mechanism (83), others suggested that sphingolipids actually promote membrane permeabilization by a graded process involving rigidification of membrane domains and subsequent creation of local structural defects $(82,84)$ (Figure 1, lower right panel). Sphingosine synthesis results from ceramide deacetylation by two ceramidases, encoded by the Asah1 and Asah2 genes, and respectively functioning at acid or neutral $\mathrm{pH}$. Notably, the expression of both enzymes is higher in DC1s than in DC2s (immgen.org), suggesting that ceramide conversion into membrane-disrupting sphingosine could be increased in DC1 endocytic compartments. This DC1-specific enrichment in sphingosine could possibly be mediated by lipid bodies, which have also been proposed to destabilize some ER or phagosomal membrane domains during their formation, thereby causing leakage of the content of these compartments (85). Along the same line, BMDCs deficient for Igtp, a GTPase controlling accumulation of lipid bodies, show a selective defect for crosspresentation (86). Moreover, intracellular accumulation of lipid droplets correlates with cross-presentation efficiency, as DC1s display significantly higher amounts of these organelles than DC2s (86). However, pharmacological interference with lipid body formation fails to influence antigen export to the cytosol in the context of saponin adjuvant-based cross-presentation (87). Thus, the precise role of lipid bodies in cross-presentation and cytosolic antigen leakage remains to be specified. 


\section{Compensatory Mechanisms for Endocytic Membrane Rupture}

Although several lines of evidence point to a contribution of endocytic membrane disruption and subsequent antigen leakage into the cytosol, this model has been repeatedly dismissed owing to its presumable lack of regulation and ensuing cell toxicity. Indeed, links between endocytic leakage and cell death were reported in different systems. Silica crystal-dependent phagosomal rupture, for example, leads to cytosolic release of intraluminal cathepsin B, which in turn activates the NLRP3 inflammasome, resulting in pyroptosis (88). Hydroxychloroquine-mediated cathepsin release from lysosomes can also trigger caspase activation and apoptosis (89), suggesting a requirement for control mechanisms to contain damaging consequences of leakage.

In this regard, the ESCRT machinery, formerly known for its key role in viral budding or cytokinetic abscission $(90,91)$, was recently identified as a core component of biological membrane repair following damage (92-96). A role for the ESCRT-I protein TSG101 in antigen export to the cytosol and cross-presentation has been previously suggested (73). However, increased cytosolic export observed following TSG101 silencing had been attributed to TSG101-dependent inhibition of MR poly-ubiquitination, required for cytosolic antigen translocation. Yet, considering the dispensable role of ubiquitination in antigen export to the cytosol (43) and the fact that TSG101 is also required for ESCRT-III-mediated repair of endolysosomal membranes (95, 96), defects in endocytic membrane repair, concomitant with TSG101 depletion, may have also contributed to the observed increased export phenotype (73). Yet, the possible involvement of ESCRT-III in controlling antigen export to the cytosol has not been investigated so far.

\section{REFERENCES}

1. Bevan MJ. Cross-priming for a secondary cytotoxic response to minor $\mathrm{H}$ antigens with H-2 congenic cells which do not cross-react in the cytotoxic assay. J Exp Med. (1976) 143:1283-8.

2. Bevan MJ. Minor $\mathrm{H}$ antigens introduced on $\mathrm{H}-2$ different stimulating cells cross-react at the cytotoxic $\mathrm{T}$ cell level during in vivo priming. J Immunol. (1976) 117:2233-8.

3. Sharma MD, Rodriguez PC, Koehn BH, Baban B, Cui Y, Guo G, et al. Activation of p53 in immature myeloid precursor cells controls differentiation into Ly6c+CD103+ monocytic antigen-presenting cells in tumors. Immunity (2018) 48:91-106.e6. doi: 10.1016/j.immuni.2017.12.014

4. Alloatti A, Rookhuizen DC, Joannas L, Carpier J-M, Iborra S, Magalhaes JG, et al. Critical role for Sec22b-dependent antigen crosspresentation in antitumor immunity. J Exp Med. (2017) 214:2231-41. doi: 10.1084/jem.20170229

5. Hildner K, Edelson BT, Purtha WE, Diamond M, Matsushita H, Kohyama M, et al. Batf3 deficiency reveals a critical role for CD8alpha+ dendritic cells in cytotoxic T cell immunity. Science (2008) 322:1097-100. doi: $10.1126 /$ science. 1164206

6. Theisen DJ, Davidson JT, Briseño CG, Gargaro M, Lauron EJ, Wang $\mathrm{Q}$, et al. WDFY4 is required for cross-presentation in response to viral and tumor antigens. Science (2018) 362:694-9. doi: 10.1126/science. aat5030

7. Roberts EW, Broz ML, Binnewies M, Headley MB, Nelson AE, Wolf DM, et al. Critical role for CD103(+)/CD141(+) dendritic cells bearing CCR7 for tumor antigen trafficking and priming of $\mathrm{T}$ cell immunity in melanoma. Cancer Cell (2016) 30:324-36. doi: 10.1016/j.ccell.2016.06.003

\section{CONCLUSION}

Identification of several critical players in antigen cross-presentation, such as Sec22b (4), or Rab43 (40), and their subsequent validation in conditional knock-out mouse pre-clinical models established a major role for this pathway in different types of immunes responses, including anti-tumor immune responses. Yet, the way antigens gain access to the cytosol during cross-presentation is far from being entirely resolved. Export to the cytosol is not only the last event in the pathway that remains largely obscure, but it is also a rate-limiting step in the process $(52,97)$. Identifying the molecular mechanism involved will certainly provide relevant targets to manipulate antigen cross-presentation for vaccination and immunotherapy purposes.

\section{AUTHOR CONTRIBUTIONS}

MG and SA designed, prepared and wrote the manuscript.

\section{FUNDING}

MG is supported by a Fondation pour la Recherche Médicale grant (grant No. FDT201805005336). MG and SA are supported by ANR-10-IDEX-0001-02 PSL*, ANR-11-LABX-0043 grants, and ERC grant 2013-AdG No. 340046 DCBIOX.

\section{ACKNOWLEDGMENTS}

The authors thank Dr. Philippe Benaroch and Dr. Marianne Burbage for critical reading and feedback on the manuscript. This work benefitted from data assembled by the Immgen consortium.

8. Song R, Harding CV. Roles of proteasomes, transporter for antigen presentation (TAP), and beta 2-microglobulin in the processing of bacterial or particulate antigens via an alternate class I MHC processing pathway. $J$ Immunol. (1996) 156:4182-90.

9. Pfeifer JD, Wick MJ, Roberts RL, Findlay K, Normark SJ, Harding CV. Phagocytic processing of bacterial antigens for class I MHC presentation to T cells. Nature (1993) 361:359-62. doi: 10.1038/361359a0

10. Harding CV, Song R. Phagocytic processing of exogenous particulate antigens by macrophages for presentation by class I MHC molecules. J Immunol. (1994) 153:4925-33.

11. Bertholet S, Goldszmid R, Morrot A, Debrabant A, Afrin F, CollazoCustodio C, et al. Leishmania antigens are presented to CD8+ T cells by a transporter associated with antigen processing-independent pathway in vitro and in vivo. J Immunol. (2006) 177:3525-33. doi: 10.4049/jimmunol.177. 6.3525

12. Shen L, Sigal LJ, Boes M, Rock KL. Important role of cathepsin S in generating peptides for TAP-independent MHC class I crosspresentation in vivo. Immunity (2004) 21:155-65. doi: 10.1016/j.immuni.2004. 07.004

13. Kovacsovics-Bankowski M, Rock KL. A phagosome-to-cytosol pathway for exogenous antigens presented on MHC class I molecules. Science (1995) 267:243-6.

14. Reis e Sousa C, Germain RN. Major histocompatibility complex class I presentation of peptides derived from soluble exogenous antigen by a subset of cells engaged in phagocytosis. J Exp Med. (1995) 182:841-51.

15. Oh YK, Harding CV, Swanson JA. The efficiency of antigen delivery from macrophage phagosomes into cytoplasm for MHC class I-restricted antigen presentation. Vaccine (1997) 15:511-8. 
16. Norbury CC, Hewlett LJ, Prescott AR, Shastri N, Watts C. Class I MHC presentation of exogenous soluble antigen via macropinocytosis in bone marrow macrophages. Immunity (1995) 3:783-91.

17. Norbury CC, Chambers BJ, Prescott AR, Ljunggren HG, Watts C. Constitutive macropinocytosis allows TAP-dependent major histocompatibility complex class I presentation of exogenous soluble antigen by bone marrow-derived dendritic cells. Eur J Immunol. (1997) 27:280-8. doi: 10.1002/eji.1830270141

18. Rodriguez A, Regnault A, Kleijmeer M, Ricciardi-Castagnoli P, Amigorena S. Selective transport of internalized antigens to the cytosol for MHC class I presentation in dendritic cells. Nat Cell Biol. (1999) 1:362-8. doi: $10.1038 / 14058$

19. Savina A, Jancic C, Hugues S, Guermonprez P, Vargas P, Moura IC, et al. NOX2 controls phagosomal $\mathrm{pH}$ to regulate antigen processing during crosspresentation by dendritic cells. Cell (2006) 126:205-18. doi: 10.1016/j.cell.2006.05.035

20. Guilliams M, Ginhoux F, Jakubzick C, Naik SH, Onai N, Schraml BU, et al. Dendritic cells, monocytes and macrophages: a unified nomenclature based on ontogeny. Nat Rev Immunol. (2014) 14:571-8. doi: 10.1038/ nri3712

21. Iyoda T, Shimoyama S, Liu K, Omatsu Y, Akiyama Y, Maeda Y, et al. The CD8+ dendritic cell subset selectively endocytoses dying cells in culture and in vivo. J Exp Med. (2002) 195:1289-302. doi: 10.1084/jem.20020161

22. den Haan JM, Lehar SM, Bevan MJ. CD8(+) but not CD8(-) dendritic cells cross-prime cytotoxic T cells in vivo. J Exp Med. (2000) 192:1685-96. doi: 10.1084/jem.192.12.1685

23. Schnorrer P, Behrens GMN, Wilson NS, Pooley JL, Smith CM, El-Sukkari D, et al. The dominant role of CD8+ dendritic cells in cross-presentation is not dictated by antigen capture. Proc Natl Acad Sci USA. (2006) 103:10729-34. doi: 10.1073/pnas.0601956103

24. Pooley JL, Heath WR, Shortman K. Cutting edge: intravenous soluble antigen is presented to CD4 T cells by CD8- dendritic cells, but cross-presented to CD8 T cells by CD8+ dendritic cells. J Immunol. (2001) 166:5327-30. doi: 10.4049/jimmunol.166.9.5327

25. Flinsenberg TWH, Compeer EB, Koning D, Klein M, Amelung FJ, van Baarle $\mathrm{D}$, et al. $\mathrm{Fc} \gamma$ receptor antigen targeting potentiates cross-presentation by human blood and lymphoid tissue BDCA-3+ dendritic cells. Blood (2012) 120:5163-72. doi: 10.1182/blood-2012-06-434498

26. Ahrens S, Zelenay S, Sancho D, Hanč P, Kjær S, Feest C, et al. Factin is an evolutionarily conserved damage-associated molecular pattern recognized by DNGR-1, a receptor for dead cells. Immunity (2012) 36:635-45. doi: 10.1016/j.immuni.2012.03.008

27. Hanč P, Fujii T, Iborra S, Yamada Y, Huotari J, Schulz O, et al. Structure of the complex of F-Actin and DNGR-1, a C-type lectin receptor involved in dendritic cell cross-presentation of dead cell-associated antigens. Immunity (2015) 42:839-49. doi: 10.1016/j.immuni.2015.04.009

28. Zelenay S, Keller AM, Whitney PG, Schraml BU, Deddouche S, Rogers NC, et al. The dendritic cell receptor DNGR-1 controls endocytic handling of necrotic cell antigens to favor cross-priming of CTLs in virus-infected mice. $J$ Clin Invest. (2012) 122:1615-27. doi: 10.1172/JCI60644

29. Sancho D, Joffre OP, Keller AM, Rogers NC, Martínez D, Hernanz-Falcón P, et al. Identification of a dendritic cell receptor that couples sensing of necrosis to immunity. Nature (2009) 458:899-903. doi: 10.1038/nature07750

30. Burgdorf S, Kautz A, Böhnert V, Knolle PA, Kurts C. Distinct pathways of antigen uptake and intracellular routing in CD4 and CD8 T cell activation. Science (2007) 316:612-6. doi: 10.1126/science.1137971

31. Savina A, Peres A, Cebrian I, Carmo N, Moita C, Hacohen N, et al. The small GTPase Rac2 controls phagosomal alkalinization and antigen crosspresentation selectively in CD8(+) dendritic cells. Immunity (2009) 30:544-55. doi: 10.1016/j.immuni.2009.01.013

32. den Haan JMM, Bevan MJ. Constitutive versus activation-dependent crosspresentation of immune complexes by $\mathrm{CD} 8(+)$ and $\mathrm{CD} 8(-)$ dendritic cells in vivo. J Exp Med. (2002) 196:817-27. doi: 10.1084/jem.200 20295

33. Neubert K, Lehmann CHK, Heger L, Baranska A, Staedtler AM, Buchholz $\mathrm{VR}$, et al. Antigen delivery to $\mathrm{CD} 11 \mathrm{c}+\mathrm{CD} 8$ - dendritic cells induces protective immune responses against experimental melanoma in mice in vivo. J Immunol. (2014) 192:5830-8. doi: 10.4049/jimmunol.13 00975

34. Chefalo PJ, Grandea AG, Van Kaer L, Harding CV. Tapasin-/- and TAP1-/macrophages are deficient in vacuolar alternate class I MHC (MHC-I) processing due to decreased MHC-I stability at phagolysosomal $\mathrm{pH}$. $J$ Immunol. (2003) 170:5825-33. doi: 10.4049/jimmunol.170.12.5825

35. Day PM, Esquivel F, Lukszo J, Bennink JR, Yewdell JW. Effect of TAP on the generation and intracellular trafficking of peptide-receptive major histocompatibility complex class I molecules. Immunity (1995) 2: 137-47.

36. Van Kaer L, Ashton-Rickardt PG, Ploegh HL, Tonegawa S. TAP1 mutant mice are deficient in antigen presentation, surface class I molecules, and CD4-8+ T cells. Cell (1992) 71:1205-14.

37. Merzougui N, Kratzer R, Saveanu L, van Endert P. A proteasome-dependent, TAP-independent pathway for cross-presentation of phagocytosed antigen. EMBO Rep. (2011) 12:1257-64. doi: 10.1038/embor.2011.203

38. Cebrian I, Visentin G, Blanchard N, Jouve M, Bobard A, Moita C, et al. Sec22b regulates phagosomal maturation and antigen crosspresentation by dendritic cells. Cell (2011) 147:1355-68. doi: 10.1016/j.cell.2011.11.021

39. Nair-Gupta P, Baccarini A, Tung N, Seyffer F, Florey O, Huang Y, et al TLR signals induce phagosomal MHC-I delivery from the endosomal recycling compartment to allow cross-presentation. Cell (2014) 158:506-21. doi: 10.1016/j.cell.2014.04.054

40. Kretzer NM, Theisen DJ, Tussiwand R, Briseño CG, Grajales-Reyes GE, Wu X, et al. RAB43 facilitates cross-presentation of cell-associated antigens by CD8 $\alpha+$ dendritic cells. J Exp Med. (2016) 213:2871-83. doi: 10.1084/jem.20160597

41. Palmowski MJ, Gileadi U, Salio M, Gallimore A, Millrain M, James E, et al. Role of immunoproteasomes in cross-presentation. J Immunol. (2006) 177:983-90. doi: 10.4049/jimmunol.177.2.983

42. Lin ML, Zhan Y, Proietto AI, Prato S, Wu L, Heath WR, et al. Selective suicide of cross-presenting CD8+ dendritic cells by cytochrome $\mathrm{c}$ injection shows functional heterogeneity within this subset. Proc Natl Acad Sci USA. (2008) 105:3029-34. doi: 10.1073/pnas.0712394105

43. Imai T, Kato Y, Kajiwara C, Mizukami S, Ishige I, Ichiyanagi T, et al. Heat shock protein 90 (HSP90) contributes to cytosolic translocation of extracellular antigen for cross-presentation by dendritic cells. Proc Natl Acad Sci USA. (2011) 108:16363-8. doi: 10.1073/pnas.1108372108

44. Mant A, Chinnery F, Elliott T, Williams AP. The pathway of cross-presentation is influenced by the particle size of phagocytosed antigen. Immunology (2012) 136:163-75. doi: 10.1111/j.1365-2567.2012.03558.x

45. Jancic C, Savina A, Wasmeier C, Tolmachova T, El-Benna J, Dang PM-C, et al. Rab27a regulates phagosomal $\mathrm{pH}$ and NADPH oxidase recruitment to dendritic cell phagosomes. Nat Cell Biol. (2007) 9:367-78. doi: $10.1038 /$ ncb 1552

46. Accapezzato D, Visco V, Francavilla V, Molette C, Donato T, Paroli M, et al. Chloroquine enhances human CD8 $+\mathrm{T}$ cell responses against soluble antigens in vivo. J Exp Med. (2005) 202:817-28. doi: 10.1084/jem.20051106

47. Gil-Torregrosa BC, Lennon-Duménil AM, Kessler B, Guermonprez P, Ploegh HL, Fruci D, et al. Control of cross-presentation during dendritic cell maturation. Eur J Immunol. (2004) 34:398-407. doi: 10.1002/eji.2003 24508

48. Zehner M, Marschall AL, Bos E, Schloetel J-G, Kreer C, Fehrenschild D, et al. The translocon protein Sec61 mediates antigen transport from endosomes in the cytosol for cross-presentation to CD8(+) T cells. Immunity (2015) 42:850-63. doi: 10.1016/j.immuni.2015.04.008

49. Lu Q, Grotzke JE, Cresswell P. A novel probe to assess cytosolic entry of exogenous proteins. Nat Commun (2018) 9:3104. doi: 10.1038/s41467-018-05556-Z

50. Burgdorf S, Schölz C, Kautz A, Tampé R, Kurts C. Spatial and mechanistic separation of cross-presentation and endogenous antigen presentation. Nat Immunol. (2008) 9:558-66. doi: 10.1038/ni.1601

51. Hotta C, Fujimaki H, Yoshinari M, Nakazawa M, Minami M. The delivery of an antigen from the endocytic compartment into the cytosol for crosspresentation is restricted to early immature dendritic cells. Immunology (2006) 117:97-107. doi: 10.1111/j.1365-2567.2005.02270.x

52. Howland SW, Wittrup KD. Antigen release kinetics in the phagosome are critical to cross-presentation efficiency. J Immunol. (2008) 180:1576-83. doi: 10.4049/jimmunol.180.3.1576

53. Houde M, Bertholet S, Gagnon E, Brunet S, Goyette G, Laplante A, et al. Phagosomes are competent organelles for antigen cross-presentation. Nature (2003) 425:402-6. doi: 10.1038/nature01912

54. Guermonprez P, Saveanu L, Kleijmeer M, Davoust J, Van Endert P, Amigorena S. ER-phagosome fusion defines an MHC class I 
cross-presentation compartment in dendritic cells. Nature (2003) 425:397-402. doi: 10.1038/nature01911

55. Gagnon E, Duclos S, Rondeau C, Chevet E, Cameron PH, SteeleMortimer $\mathrm{O}$, et al. Endoplasmic reticulum-mediated phagocytosis is a mechanism of entry into macrophages. Cell (2002) 110:119-31. doi: 10.1016/S0092-8674(02)00797-3

56. Touret N, Paroutis P, Terebiznik M, Harrison RE, Trombetta S, Pypaert M, et al. Quantitative and dynamic assessment of the contribution of the ER to phagosome formation. Cell (2005) 123:157-70. doi: 10.1016/j.cell.2005. 08.018

57. Kato Y, Kajiwara C, Ishige I, Mizukami S, Yamazaki C, Eikawa S, et al. HSP70 and HSP90 differentially regulate translocation of extracellular antigen to the cytosol for cross-presentation. Autoimmune Dis. (2012) 2012:745962. doi: 10.1155/2012/745962

58. Singh R, Cresswell P. Defective cross-presentation of viral antigens in GILTfree mice. Science (2010) 328:1394-8. doi: 10.1126/science.1189176

59. Imai J, Hasegawa H, Maruya M, Koyasu S, Yahara I. Exogenous antigens are processed through the endoplasmic reticulum-associated degradation (ERAD) in cross-presentation by dendritic cells. Int Immunol. (2005) 17:4553. doi: 10.1093/intimm/dxh184

60. Schäuble N, Cavalié A, Zimmermann R, Jung M. Interaction of Pseudomonas aeruginosa exotoxin A with the human Sec61 complex suppresses passive calcium efflux from the endoplasmic reticulum. Channels Austin Tex. (2014) 8:76-83. doi: 10.4161/chan.26526

61. Koopmann JO, Albring J, Hüter E, Bulbuc N, Spee P, Neefjes J, et al. Export of antigenic peptides from the endoplasmic reticulum intersects with retrograde protein translocation through the Sec61p channel. Immunity (2000) 13:117-27. doi: 10.1016/S1074-7613(00)00013-3

62. Ackerman AL, Giodini A, Cresswell P. A role for the endoplasmic reticulum protein retrotranslocation machinery during crosspresentation by dendritic cells. Immunity (2006) 25:607-17. doi: 10.1016/j.immuni.2006.08.017

63. Goldszmid RS, Coppens I, Lev A, Caspar P, Mellman I, Sher A. Host ERparasitophorous vacuole interaction provides a route of entry for antigen cross-presentation in Toxoplasma gondii-infected dendritic cells. J Exp Med. (2009) 206:399-410. doi: 10.1084/jem.20082108

64. Dudziak D, Kamphorst AO, Heidkamp GF, Buchholz VR, Trumpfheller C, Yamazaki S, et al. Differential antigen processing by dendritic cell subsets in vivo. Science (2007) 315:107-11. doi: 10.1126/science.1136080

65. Römisch K. A case for Sec61 channel involvement in ERAD. Trends Biochem Sci. (2017) 42:171-9. doi: 10.1016/j.tibs.2016.10.005

66. Grotzke JE, Kozik P, Morel J-D, Impens F, Pietrosemoli N, Cresswell P, et al. Sec61 blockade by mycolactone inhibits antigen cross-presentation independently of endosome-to-cytosol export. Proc Natl Acad Sci USA. (2017) 114:E5910-19. doi: 10.1073/pnas.1705242114

67. Schoebel S, Mi W, Stein A, Ovchinnikov S, Pavlovicz R, DiMaio F, et al. Cryo-EM structure of the protein-conducting ERAD channel Hrd1 in complex with Hrd3. Nature (2017) 548:352-5. doi: 10. 1038/nature23314

68. Stein A, Ruggiano A, Carvalho P, Rapoport TA. Key steps in ERAD of luminal ER proteins reconstituted with purified components. Cell (2014) 158:1375-88. doi: 10.1016/j.cell.2014.07.050

69. Mehnert M, Sommer T, Jarosch E. Der1 promotes movement of misfolded proteins through the endoplasmic reticulum membrane. Nat Cell Biol. (2014) 16:77-86. doi: 10.1038/ncb2882

70. Kadowaki H, Satrimafitrah P, Takami Y, Nishitoh H. Molecular mechanism of ER stress-induced pre-emptive quality control involving association of the translocon, Derlin-1, and HRD1. Sci Rep. (2018) 8:7317. doi: 10.1038/s41598-018-25724-x

71. Ménager J, Ebstein F, Oger R, Hulin P, Nedellec S, Duverger E, et al. Crosspresentation of synthetic long peptides by human dendritic cells: a process dependent on ERAD component p97/VCP but Not sec61 and/or Derlin-1. PLoS ONE (2014) 9:e89897. doi: 10.1371/journal.pone.0089897

72. Ye Y, Meyer HH, Rapoport TA. Function of the p97-Ufd1-Npl4 complex in retrotranslocation from the ER to the cytosol: dual recognition of nonubiquitinated polypeptide segments and polyubiquitin chains. J Cell Biol. (2003) 162:71-84. doi: 10.1083/jcb.200302169

73. Zehner M, Chasan AI, Schuette V, Embgenbroich M, Quast T, Kolanus W, et al. Mannose receptor polyubiquitination regulates endosomal recruitment of p97 and cytosolic antigen translocation for cross-presentation. Proc Natl Acad Sci USA. (2011) 108:9933-8. doi: 10.1073/pnas.1102397108

74. Giodini A, Cresswell P. Hsp90-mediated cytosolic refolding of exogenous proteins internalized by dendritic cells. EMBO J. (2008) 27:201-11. doi: 10.1038/sj.emboj.7601941

75. Akishiba M, Takeuchi T, Kawaguchi Y, Sakamoto K, Yu H-H, Nakase I, et al. Cytosolic antibody delivery by lipid-sensitive endosomolytic peptide. Nat Chem. (2017) 9:751-61. doi: 10.1038/nchem.2779

76. El-Sayed A, Futaki S, Harashima H. Delivery of macromolecules using arginine-rich cell-penetrating peptides: ways to overcome endosomal entrapment. AAPS J. (2009) 11:13-22. doi: 10.1208/s12248-008-9071-2

77. Li M, Tao Y, Shu Y, LaRochelle JR, Steinauer A, Thompson D, et al. Discovery and characterization of a peptide that enhances endosomal escape of delivered proteins in vitro and in vivo. J Am Chem Soc. (2015) 137:1408493. doi: 10.1021/jacs.5b05694

78. Dingjan I, Verboogen DR, Paardekooper LM, Revelo NH, Sittig SP, Visser LJ, et al. Lipid peroxidation causes endosomal antigen release for cross-presentation. Sci Rep. (2016) 6:22064. doi: 10. 1038/srep22064

79. Dingjan I, Paardekooper LM, Verboogen DRJ, von Mollard GF, Ter Beest M, van den Bogaart G. VAMP8-mediated NOX2 recruitment to endosomes is necessary for antigen release. Eur J Cell Biol. (2017) 96:705-14. doi: 10.1016/j.ejcb.2017.06.007

80. Wong-Ekkabut J, Xu Z, Triampo W, Tang I-M, Tieleman DP, Monticelli L. Effect of lipid peroxidation on the properties of lipid bilayers: a molecular dynamics study. Biophys J. (2007) 93:4225-36. doi: 10.1529/biophysj.107.112565

81. Agmon E, Solon J, Bassereau P, Stockwell BR. Modeling the effects of lipid peroxidation during ferroptosis on membrane properties. Sci Rep. (2018) 8:5155. doi: 10.1038/s41598-018-23408-0

82. Contreras F-X, Sot J, Alonso A, Goñi FM. Sphingosine increases the permeability of model and cell membranes. Biophys J. (2006) 90:4085-92. doi: 10.1529/biophysj.105.076471

83. Siskind LJ, Kolesnick RN, Colombini M. Ceramide channels increase the permeability of the mitochondrial outer membrane to small proteins. J Biol Chem. (2002) 277:26796-803. doi: 10.1074/jbc.M2007 54200

84. Jiménez-Rojo N, Sot J, Viguera AR, Collado MI, Torrecillas A, GómezFernández JC, et al. Membrane permeabilization induced by sphingosine: effect of negatively charged lipids. Biophys J. (2014) 106:2577-84. doi: 10.1016/j.bpj.2014.04.038

85. Ploegh HL. A lipid-based model for the creation of an escape hatch from the endoplasmic reticulum. Nature (2007) 448:435-8. doi: 10 . 1038/nature06004

86. Bougnères L, Helft J, Tiwari S, Vargas $\mathrm{P}$, Chang BH-J, Chan L, et al. A role for lipid bodies in the cross-presentation of phagocytosed antigens by MHC class I in dendritic cells. Immunity (2009) 31:232-44. doi: 10.1016/j.immuni.2009.06.022

87. den Brok MH, Büll C, Wassink M, de Graaf AM, Wagenaars JA, Minderman $M$, et al. Saponin-based adjuvants induce cross-presentation in dendritic cells by intracellular lipid body formation. Nat Commun. (2016) 7:13324. doi: $10.1038 /$ ncomms13324

88. Hornung V, Bauernfeind F, Halle A, Samstad EO, Kono H, Rock $\mathrm{KL}$, et al. Silica crystals and aluminum salts activate the NALP3 inflammasome through phagosomal destabilization. Nat Immunol. (2008) 9:847-56. doi: 10.1038/ni.1631

89. Boya P, Gonzalez-Polo R-A, Poncet D, Andreau K, Vieira HLA, Roumier $\mathrm{T}$, et al. Mitochondrial membrane permeabilization is a critical step of lysosome-initiated apoptosis induced by hydroxychloroquine. Oncogene (2003) 22:3927-36. doi: 10.1038/sj.onc.1206622

90. Carlton JG, Caballe A, Agromayor M, Kloc M, Martin-Serrano J. ESCRT-III governs the Aurora B-mediated abscission checkpoint through CHMP4C. Science (2012) 336:220-5. doi: 10.1126/science. 1217180

91. Garrus JE, von Schwedler UK, Pornillos OW, Morham SG, Zavitz KH, Wang $\mathrm{HE}$, et al. Tsg101 and the vacuolar protein sorting pathway are essential for HIV-1 budding. Cell (2001) 107:55-65. doi: 10.1016/S0092-8674(01) 00506-2 
92. Olmos Y, Hodgson L, Mantell J, Verkade P, Carlton JG. ESCRTIII controls nuclear envelope reformation. Nature (2015) 522:236-9. doi: 10.1038/nature14503

93. Vietri M, Schink KO, Campsteijn C, Wegner CS, Schultz SW, Christ L, et al. Spastin and ESCRT-III coordinate mitotic spindle disassembly and nuclear envelope sealing. Nature (2015) 522:231-5. doi: 10.1038/nature14408

94. Jimenez AJ, Maiuri P, Lafaurie-Janvore J, Divoux S, Piel M, Perez F. ESCRT machinery is required for plasma membrane repair. Science (2014) 343:1247136. doi: 10.1126/science. 1247136

95. Skowyra ML, Schlesinger PH, Naismith TV, Hanson PI. Triggered recruitment of ESCRT machinery promotes endolysosomal repair. Science (2018) 360:eaar5078. doi: 10.1126/science.aar5078

96. Radulovic M, Schink KO, Wenzel EM, Nähse V, Bongiovanni A, Lafont F, et al. ESCRT-mediated lysosome repair precedes lysophagy and promotes cell survival. EMBO J. (2018) 37:e99753. doi: 10.15252/embj.201899753
97. Moore MW, Carbone FR, Bevan MJ. Introduction of soluble protein into the class I pathway of antigen processing and presentation. Cell (1988) 54:777-85.

Conflict of Interest Statement: The authors declare that the research was conducted in the absence of any commercial or financial relationships that could be construed as a potential conflict of interest.

Copyright $\odot 2019$ Gros and Amigorena. This is an open-access article distributed under the terms of the Creative Commons Attribution License (CC BY). The use, distribution or reproduction in other forums is permitted, provided the original author(s) and the copyright owner(s) are credited and that the original publication in this journal is cited, in accordance with accepted academic practice. No use, distribution or reproduction is permitted which does not comply with these terms. 